\title{
A Novel Value for the Parameter in the Dai-Liao-Type Conjugate Gradient Method
}

\author{
Branislav Ivanov $\left(\mathbb{D},{ }^{1}\right.$ Predrag S. Stanimirović $\mathbb{D}^{2},{ }^{2}$ Bilall I. Shaini, ${ }^{3}$ Hijaz Ahmad $\left(\mathbb{D},{ }^{4}\right.$ \\ and Miao-Kun Wang $\mathbb{D}^{5}$
}

${ }^{1}$ Technical Faculty in Bor, University of Belgrade, Vojske Jugoslavije 12, 19210 Bor, Serbia

${ }^{2}$ Faculty of Sciences and Mathematics, University of Niš, Višegradska 33, 18000 Niš, Serbia

${ }^{3}$ University of Tetovo, St. Ilinden, n.n., Tetovo, North Macedonia

${ }^{4}$ Department of Basic Sciences, University of Engineering and Technology Peshawar, Pakistan

${ }^{5}$ Department of Mathematics, Huzhou University, Huzhou 313000, China

Correspondence should be addressed to Miao-Kun Wang; wmk000@126.com

Received 29 October 2020; Revised 28 November 2020; Accepted 15 January 2021; Published 30 January 2021

Academic Editor: Ioan Rasa

Copyright (c) 2021 Branislav Ivanov et al. This is an open access article distributed under the Creative Commons Attribution License, which permits unrestricted use, distribution, and reproduction in any medium, provided the original work is properly cited.

\begin{abstract}
A new rule for calculating the parameter $t$ involved in each iteration of the MHSDL (Dai-Liao) conjugate gradient (CG) method is presented. The new value of the parameter initiates a more efficient and robust variant of the Dai-Liao algorithm. Under proper conditions, theoretical analysis reveals that the proposed method in conjunction with backtracking line search is of global convergence. Numerical experiments are also presented, which confirm the influence of the new value of the parameter $t$ on the behavior of the underlying CG optimization method. Numerical comparisons and the analysis of obtained results considering Dolan and Morés performance profile show better performances of the novel method with respect to all three analyzed characteristics: number of iterative steps, number of function evaluations, and CPU time.
\end{abstract}

\section{Introduction and Background Results}

The topic of our research is solving the unconstrained nonlinear optimization problem

$$
\min f(x), \quad x \in \mathbb{R}^{n},
$$

where the function $f: \mathbb{R}^{n} \longrightarrow \mathbb{R}$ is continuously differentiable and bounded below. Following the standard notation, $g_{k}=\nabla f\left(x_{k}\right)$ denotes the gradient, $s_{k-1}=x_{k}-x_{k-1}$ and $y_{k-1}$ $=g_{k}-g_{k-1}$. Using an extended conjugacy condition

$$
d_{k}^{\mathrm{T}} y_{k-1}=-t g_{k}^{\mathrm{T}} s_{k-1}, \quad t>0,
$$

Dai and Liao in [1] proposed the conjugate gradient (CG) method

$$
x_{k+1}=x_{k}+\alpha_{k} d_{k} \text {, }
$$

where the step size $\alpha_{k}$ is a positive parameter, $x_{k}$ is an already generated point, $x_{k+1}$ is a new iterative point, and $d_{k}$ is a suitable search direction. The search directions $d_{k}$ are generated by the conceptual formula

$$
d_{k}= \begin{cases}-g_{0}, & k=0, \\ -g_{k}+\beta_{k}^{\mathrm{DL}} d_{k-1}, & k \geq 1,\end{cases}
$$

where the conjugate gradient coefficient $\beta_{k}^{\mathrm{DL}}$ is defined by

$$
\beta_{k}^{\mathrm{DL}}=Y(t):=\frac{g_{k}^{\mathrm{T}} y_{k-1}}{d_{k-1}^{\mathrm{T}} y_{k-1}}-t \frac{g_{k}^{\mathrm{T}} s_{k-1}}{d_{k-1}^{\mathrm{T}} y_{k-1}}, \quad t>0,
$$

wherein $t>0$ is a scalar.

Some well-known formulas for defining $\beta_{k}$ have been created by modifying the conjugate gradient parameter $\beta_{k}^{\mathrm{DL}}[2-9]$. One of them is denoted as $\beta_{k}^{\mathrm{MHSDL}}$ and defined 
The backtracking line search.

Require: Nonlinear objective function $f(x)$, search direction $d_{k}$, previous point $x_{k}$, and real quantities $0<\omega<0.5$ and $\varphi \in(0,1)$.

1: $\alpha=1$.

2: While $f\left(x_{k}+\alpha d_{k}\right)>f\left(x_{k}\right)+\omega \alpha g_{k}^{\mathrm{T}} d_{k}$, do $\alpha:=\alpha \varphi$.

3: Return $\alpha_{k}=\alpha$.

Algorithm 1:

in [7] by

$$
\beta_{k}^{\mathrm{MHSDL}}=Y_{1}(t):=\frac{g_{k}^{\mathrm{T}} \widehat{y_{k-1}}}{d_{k-1}^{\mathrm{T}} y_{k-1}}-t \frac{g_{k}^{\mathrm{T}} s_{k-1}}{d_{k-1}^{\mathrm{T}} y_{k-1}},
$$

where $t>0$ is a scalar as in (5) and $\widehat{y_{k-1}}=g_{k}-\left(\left\|g_{k}\right\| / \|\right.$ $\left.g_{k-1} \|\right) g_{k-1}$.

The family of CG methods for nonlinear optimization has reached great popularity lately, thanks to the various benefits and advantages it possesses. The most important property is based on computationally efficient iterations arising from a simple CG rule. This property initiates the high efficiency of CG methods with respect to analogous methods for nonlinear optimization. Moreover, global convergence is ensured under suitable conditions. Finally, the application of various CG methods in solving image restoration problems has become an important research topic $[10,11]$.

Since the parameter $t$ is important for the numerical behavior of Dai-Liao (DL) CG methods [12], one of the most important problems in the implementation of the DL class CG method is to determine a proper value $t>0$ which will give desirable results. Many scientists have invested a lot of time and effort in the previous period to determine the best definition of the nonnegative parameter $t$ in the DL class CG methods. So far, the research in finding the appropriate value of $t$ has evolved in two directions. One group of methods is aimed at finding an appropriate fixed value for $t$ $[1,2,6-8]$, while methods from another group promote appropriate rules for computing values of $t$ in each iteration, which ensure a satisfactory decrease of the objective. In our research, we will pay attention to the second research stream: find the parameter $t$ whose values change through iterations so that the faster convergence is achieved. The value of the parameter $t$ defined in the $k$ th iteration will be denoted by $t$ $(k):=t_{k}$.

In order to complete the presentation, we will restate the main principles proposed so far for computing $t_{k}$. Hager and Zhang in $[13,14]$ proposed the DL CG method (5), known as CG-DESCENT, where $t(k) \equiv t_{k 1}$ is defined by

$$
t(k) \equiv t_{k 1}:=2 \frac{\left\|y_{k-1}\right\|^{2}}{y_{k-1}^{\mathrm{T}} s_{k-1}} .
$$

Dai and Kou [15] suggested the conjugate gradient coef- ficient $\beta_{k}^{\mathrm{DK}}$ of the form

$\beta_{k}^{\mathrm{DK}}=Y\left(\tau_{k}+\frac{\left\|y_{k-1}\right\|^{2}}{y_{k-1}^{\mathrm{T}} s_{k-1}}-\frac{y_{k-1}^{\mathrm{T}} s_{k-1}}{\left\|s_{k-1}\right\|^{2}}\right)=\frac{g_{k}^{\mathrm{T}} y_{k-1}}{y_{k-1}^{\mathrm{T}} d_{k-1}}-\left(\tau_{k}+\frac{\left\|y_{k-1}\right\|^{2}}{y_{k-1}^{\mathrm{T}} s_{k-1}}-\frac{y_{k-1}^{\mathrm{T}} s_{k-1}}{\left\|s_{k-1}\right\|^{2}}\right) \frac{g_{k}^{\mathrm{T}} s_{k-1}}{d_{k-1}^{\mathrm{T}} y_{k-1}}$,

where $\tau_{k}$ is the scaling parameter arising from the self-scaling memoryless BFGS method. Clearly, the Dai and Kou (DK) method is a member of the DL class CG methods, which is determined by

$$
t(k) \equiv t_{k 2}:=\tau_{k}+\frac{\left\|y_{k-1}\right\|^{2}}{y_{k-1}^{\mathrm{T}} s_{k-1}}-\frac{y_{k-1}^{\mathrm{T}} s_{k-1}}{\left\|s_{k-1}\right\|^{2}} .
$$

The results given in [15] confirm that the DK iterations outperform many existing CG methods. Following the development of DL methods, Babaie-Kafaki and Ghanbari [16] defined two new ways to calculate the value of the parameter $t$ in (5), as in the following two formulas:

$$
\begin{gathered}
t(k) \equiv t_{k 3}:=\frac{s_{k-1}^{\mathrm{T}} y_{k-1}}{\left\|s_{k-1}\right\|^{2}}+\frac{\left\|y_{k-1}\right\|}{\left\|s_{k-1}\right\|}, \\
t(k) \equiv t_{k 4}:=\frac{\left\|y_{k-1}\right\|}{\left\|s_{k-1}\right\|} .
\end{gathered}
$$

Andrei in [17] proposed the new rule for calculating $t$ in order to define $Y(t)$ in (5) and defined a new variant of the DL class CG methods, denoted by DLE, with

$$
t(k) \equiv t_{k 5}:=\frac{s_{k-1}^{\mathrm{T}} y_{k-1}}{\left\|s_{k-1}\right\|^{2}}
$$

Lotfi and Hosseini in [18] proposed the following rule for determining the parameter $t(k)$, using the expression

$$
t(k) \equiv t_{k 6}:=\max \left\{t_{k 6}^{*}, v \frac{\left\|y_{k-1}\right\|^{2}}{s_{k-1}^{T} y_{k-1}}\right\}
$$




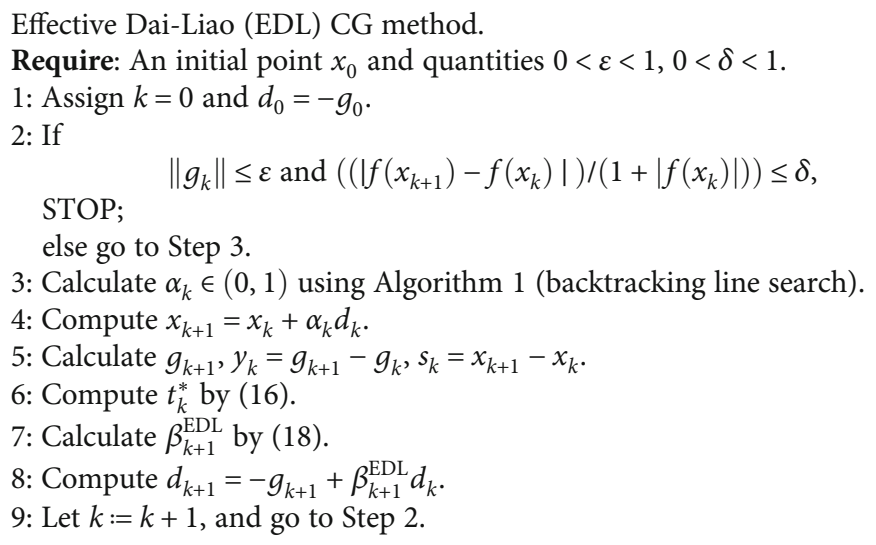

Algorithm 2:

where

$$
\begin{gathered}
t_{k 6}^{*}:=\frac{\left(1-h_{k}\left\|g_{k-1^{r}}\right\|\right) s_{k-1}^{\mathrm{T}} g_{k}+\left(g_{k}^{\mathrm{T}} y_{k-1} / y_{k-1}^{\mathrm{T}} s_{k-1}\right) h_{k}\left\|g_{k-1}\right\|^{r}\left\|s_{k-1}\right\|^{2}}{g_{k}^{\mathrm{T}} s_{k-1}+\left(g_{k}^{\mathrm{T}} s_{k-1} / s_{k-1}^{\mathrm{T}} y_{k-1}\right) h_{k}\left\|g_{k-1}\right\|^{r}\left\|s_{k-1}\right\|^{2}}, \\
h_{k}=C+\max \left\{-\frac{s_{k-1}^{\mathrm{T}} y_{k-1}}{\left\|s_{k-1}\right\|^{2}}, 0\right\}\left\|g_{k-1}\right\|^{-r},
\end{gathered}
$$

and $v>1 / 4, C$, and $r$ are three positive constants.

On the basis of the above overview of the main CG methods and motivated by the strong theoretical properties and computational efficiency of modified Dai-Liao CG methods proposed by many researchers, we suggest a new way of calculating the value of the parameter $t(k)$. As a consequence, the corresponding CG method of DL type, termed as the Effective Dai-Liao (EDL) method, is proposed and its convergence is proven. Numerical testing and comparison with other known DL variants are presented in order to show the effectiveness of the introduced method. Analysis of generated numerical results exhibits that the proposed EDL method is efficient compared with other DL-type methods.

The global organization of sections is described as follows. Introduction, motivation, and a brief overview of the preliminary results are given in Section 1. A new rule for calculating the variable parameter $t(k)$ is proposed in Section 2. An effective algorithm and global convergence of the EDL method initiated by $t(k)$ are given in the same section. The new EDL method is tested in Section 3 on some unlimited optimization test problems and compared against some known variants of the DL class methods. Finally, concluding remarks are presented in the last concluding section.

\section{A Modified Dai-Liao Method and Its Convergence}

Popularity in defining new rules for calculating $t(k)$ is a guarantee that such an approach is effective and still insufficiently explored. The idea for defining a new parameter $t_{k}^{*}$ comes from previously described rules for computing $t(k)$, particularly from the paper Li and Ruan [19] and from the idea which can be found in the paper Yuan et al. [11]. Further, analyzing the results from $[1,2,6-8]$, we conclude that the scalar $t$ was defined by a fixed value of 0.1 in related numerical experiments. Also, numerical experience related to the fixed valued $t=1$ was reported in [1]. According to this experience, our intention is to define variable values $t(k)$ inside the interval $(0,1)$.

To successfully define $t(k)$ with values belonging to the interval $(0,1)$, let us start from the definition of the quantity $L_{k}$ which was used in defining the direction $d_{k}$ in [19]. The parameter $L_{k}$ was defined by $L_{k}=s_{k-1}^{\mathrm{T}} s_{k-1} / s_{k-1}^{\mathrm{T}} y_{k-1}^{*} \in(0,1), k$ $\geq 0$, where

$$
y_{k-1}^{*}=y_{k-1}+\left(\max \left\{0,-\frac{s_{k-1}^{\mathrm{T}} y_{k-1}}{\left\|s_{k-1}\right\|^{2}}\right\}+1\right) s_{k-1} .
$$

By putting $\mathrm{y}_{k-1}^{*}$ into $L_{k}$, the following can be obtained:

$$
\begin{aligned}
L_{k} & =\frac{s_{k-1}^{\mathrm{T}} s_{k-1}}{s_{k-1}^{\mathrm{T}}\left(y_{k-1}+\left(\max \left\{0,-\left(s_{k-1}^{\mathrm{T}} y_{k-1} /\left\|s_{k-1}\right\|^{2}\right)\right\}+1\right) s_{k-1}\right)} \\
& =\frac{\left\|s_{k-1}\right\|^{2}}{s_{k-1}^{\mathrm{T}} y_{k-1}+\left(\max \left\{0,-\left(s_{k-1}^{\mathrm{T}} y_{k-1} /\left\|s_{k-1}\right\|^{2}\right)\right\}+1\right)\left\|s_{k-1}\right\|^{2}} .
\end{aligned}
$$

Further, with certain modifications and substitutions in the equation defining $L_{k}$, as well as using the function max, which chooses the maximum between the value of the expression $d_{k-1}^{\mathrm{T}} g_{k}$ and 1 , we come to a new definition of the parameter $t(k)$. As described in advance imposed desired restrictions, the novel parameter $t_{k}^{*}$ is defined by

$$
t_{k}^{*}=\frac{\left\|g_{k}\right\|^{2}}{\max \left\{1, d_{k-1}^{\mathrm{T}} g_{k}\right\}+\left(\max \left\{0,\left(d_{k-1}^{\mathrm{T}} g_{k} /\left\|g_{k}\right\|^{2}\right)\right\}+1\right)\left\|g_{k}\right\|^{2}}
$$


TABLE 1: Summary results of EDL, MHSDL3, MHSDL4, MHSDL5, and MHSDL6 methods with respect to NI.

\begin{tabular}{|c|c|c|c|c|c|}
\hline Test function & MHSDL3 & MHSDL4 & MHSDL5 & EDL & MHSDL6 \\
\hline Extended penalty & 1466 & 2243 & 2231 & 1259 & 1371 \\
\hline Perturbed quadratic & 1203710 & 754291 & 746557 & 305622 & 423037 \\
\hline Raydan 1 & 159055 & 110587 & 106586 & 55477 & 75154 \\
\hline Raydan 2 & 1636 & 441 & 441 & 70 & 209 \\
\hline Diagonal 1 & 116788 & 78844 & 73512 & 30978 & 20332 \\
\hline Diagonal 2 & 176983 & 270434 & 271595 & 515000 & 271295 \\
\hline Diagonal 3 & 150328 & 98647 & 104417 & 47155 & 37711 \\
\hline Hager & 8666 & 5219 & 5157 & 3234 & 3625 \\
\hline Generalized tridiagonal 1 & 1862 & 1471 & 1485 & 639 & 877 \\
\hline Extended TET & 1357 & 5954 & 5915 & 4030 & 2664 \\
\hline Diagonal 4 & 30693 & 19589 & 19332 & 8040 & 12012 \\
\hline Diagonal 5 & 1721 & 25120 & 25120 & 60 & 216 \\
\hline Extended Himmelblau & 1777 & 8023 & 7946 & 1376 & 3682 \\
\hline Perturbed quadratic diagonal & 2940970 & 2115659 & 2027128 & 1136414 & 1352704 \\
\hline Quadratic QF1 & 1270802 & 799192 & 786032 & 309509 & 325415 \\
\hline Extended quadratic penalty QP1 & 770 & 594 & 575 & 560 & 543 \\
\hline Extended quadratic penalty QP2 & 399671 & 240530 & 245254 & 96620 & 137799 \\
\hline Extended quadratic exponential EP1 & 462 & 606 & 606 & 513 & 526 \\
\hline Extended tridiagonal 2 & 3119 & 2176 & 2177 & 1132 & 1455 \\
\hline ARWHEAD (CUTE) & 88824 & 69868 & 67413 & 40713 & 48669 \\
\hline ENGVAL1 (CUTE) & 2323 & 1407 & 1415 & 552 & 820 \\
\hline INDEF (CUTE) & 20 & 31 & 1080 & 23 & 36240 \\
\hline QUARTC (CUTE) & 173913 & 262291 & 262291 & 524299 & 262181 \\
\hline Diagonal 6 & 1824 & 508 & 508 & 70 & 227 \\
\hline Generalized quartic & 1208 & 1403 & 2846 & 1265 & 1154 \\
\hline Diagonal 7 & 3217 & 655 & 655 & 653 & 580 \\
\hline Diagonal 8 & 511 & 698 & 698 & 686 & 596 \\
\hline Full Hessian FH3 & 1456 & 5353 & 5350 & 2523 & 3176 \\
\hline
\end{tabular}

It is easy to verify that $t_{k}^{*}$ defined by (16) satisfies

$$
0<t_{k}^{*} \leq \frac{\left\|g_{k}\right\|^{2}}{1+(0+1)\left\|g_{k}\right\|^{2}}=\frac{\left\|g_{k}\right\|^{2}}{1+\left\|g_{k}\right\|^{2}}<1
$$

Accordingly, $t_{k}^{*} \in(0,1)$, which was our initial intention. Clearly, greater values of $\left\|g_{k}\right\|$ lead to values $t_{k}^{*} \nearrow 1$. Further, since the trend $\left\|g_{k}\right\| \longrightarrow 0$ is expectable, we can expect smaller values $t_{k}^{*} \searrow 0$ in late iterations. Therefore, $t_{k}^{*}$ is suitable for defining corresponding conjugate gradient coefficient $Y(t)$ or $Y_{1}(t)$ and further DL CG iterations (4).

Considering $t=t_{k}^{*}$ in (6), it is reasonable to propose a novel variant of the Dai-Liao CG parameter $\beta_{k}^{\mathrm{EDL}}$ which is subject to the following rule during the iterative process:

$$
\beta_{k}^{\mathrm{EDL}}=Y_{1}\left(t_{k}^{*}\right):=\frac{\left\|g_{k}\right\|^{2}-\left(\left\|g_{k}\right\| /\left\|g_{k-1}\right\|\right)\left|g_{k}^{\mathrm{T}} g_{k-1}\right|}{d_{k-1}^{\mathrm{T}} y_{k-1}}-t_{k}^{*} \frac{g_{k}^{\mathrm{T}} s_{k-1}}{d_{k-1}^{\mathrm{T}} y_{k-1}} .
$$

Before the main algorithm, it is necessary to define the backtracking line search as one of the most popular and practical methods for computing the step length $\alpha_{k}$ in (3). The procedure for the backtracking line search proposed in [20] starts from the initial value $\alpha=1$ and generates output values which ensure that the goal function decreases in each iteration. Consequently, it is appropriate to use Algorithm 1, restated from [21], in order to determine the primary step size $\alpha_{k}$.

Algorithm 2 describes a computational framework for the EDL method.

It is necessary to examine the properties of the EDL method and prove its convergence.

\section{Assumption 1.}

(1) The level set $\mathscr{M}=\left\{x \in \mathbb{R}^{n} \mid f(x) \leq f\left(x_{0}\right)\right\}$, defined upon the initial point $x_{0}$ of the iterative method (3), is bounded.

(2) The goal function $f$ is continuous and differentiable in a neighborhood $\mathscr{P}$ of $M$ with the Lipschitz 
TABLE 2: Summary results of EDL, MHSDL3, MHSDL4, MHSDL5, and MHSDL6 methods with respect to NFE.

\begin{tabular}{|c|c|c|c|c|c|}
\hline Test function & MHSDL3 & MHSDL4 & MHSDL5 & EDL & MHSDL6 \\
\hline Extended penalty & 54876 & 73764 & 73429 & 46820 & 49791 \\
\hline Perturbed quadratic & 56691737 & 34287604 & 33885701 & 13168688 & 18486375 \\
\hline Raydan 1 & 5066739 & 3364983 & 3236335 & 1551846 & 2170553 \\
\hline Raydan 2 & 6554 & 1162 & 1162 & 159 & 428 \\
\hline Diagonal 1 & 5004640 & 3256274 & 3022015 & 1200086 & 744278 \\
\hline Diagonal 2 & 353976 & 540878 & 543200 & 1030010 & 542600 \\
\hline Diagonal 3 & 6339146 & 3998904 & 4229565 & 1798032 & 1400076 \\
\hline Hager & 192474 & 107413 & 106534 & 59187 & 69735 \\
\hline Generalized tridiagonal 1 & 37429 & 27860 & 28138 & 10760 & 15177 \\
\hline Extended TET & 19546 & 77422 & 76925 & 40340 & 29334 \\
\hline Diagonal 4 & 713120 & 425023 & 418666 & 155027 & 242443 \\
\hline Diagonal 5 & 6874 & 50460 & 50460 & 140 & 442 \\
\hline Extended Himmelblau & 45972 & 192362 & 190524 & 26104 & 80854 \\
\hline Perturbed quadratic diagonal & 135901222 & 94177165 & 90238441 & 48147512 & 57702654 \\
\hline Quadratic QF1 & 55972697 & 33836473 & 33243711 & 12316721 & 12853424 \\
\hline Extended quadratic penalty QP1 & 17016 & 12882 & 12565 & 11116 & 10544 \\
\hline Extended quadratic penalty QP2 & 13015888 & 7454686 & 7584960 & 2743358 & 4030601 \\
\hline Extended quadratic exponential EP1 & 14914 & 18463 & 18463 & 14132 & 15133 \\
\hline Extended tridiagonal 2 & 36450 & 22564 & 22379 & 9687 & 12920 \\
\hline ARWHEAD (CUTE) & 4296028 & 3305257 & 3182138 & 1846606 & 2230650 \\
\hline ENGVAL1 (CUTE) & 40462 & 22432 & 22898 & 8209 & 12858 \\
\hline INDEF (CUTE) & 1808 & 2182 & 5995 & 2060 & 104962 \\
\hline QUARTC (CUTE) & 347926 & 524662 & 524662 & 1048648 & 524422 \\
\hline Diagonal 6 & 7394 & 1416 & 1408 & 159 & 468 \\
\hline Generalized quartic & 14364 & 21842 & 48770 & 16695 & 14103 \\
\hline Diagonal 7 & 6454 & 6838 & 6838 & 3891 & 4521 \\
\hline Diagonal 8 & 6098 & 6938 & 6938 & 4161 & 5494 \\
\hline Full Hessian FH3 & 60792 & 212799 & 212701 & 89890 & 114962 \\
\hline
\end{tabular}

continuous gradient $g$. This assumption implies the existence of a positive constant $L>0$ satisfying

$$
\|g(u)-g(v)\| \leq L\|u-v\|, \quad \forall u, v \in \mathscr{P} .
$$

Assumption 1 initiates the existence of positive constants $D$ and $\gamma$ satisfying

$$
\begin{gathered}
\|u-v\| \leq D, \quad \forall u, v \in \mathscr{P} \\
\|g(u)\| \leq \gamma, \quad \forall u \in \mathscr{P} .
\end{gathered}
$$

The conditions from Assumption 1 are assumed. In view of the uniform convexity of $f$, there is a constant $\theta>0$ that satisfies

$$
(g(u)-g(v))^{\mathrm{T}}(u-v) \geq \theta\|u-v\|^{2}, \quad \text { for all } u, v \in \mathscr{M},
$$

or equivalently,

$$
f(u) \geq f(v)+g(v)^{\mathrm{T}}(u-v)+\frac{\theta}{2}\|u-v\|^{2}, \quad \text { for all } u, v \in \mathscr{M} .
$$

It follows from (21) and (22) that

$$
\begin{gathered}
s_{k-1}^{\mathrm{T}} y_{k-1} \geq \theta\left\|s_{k-1}\right\|^{2}, \\
f\left(x_{k-1}\right)-f\left(x_{k}\right) \geq-g\left(x_{k}\right)^{\mathrm{T}} s_{k-1}+\frac{\theta}{2}\left\|s_{k-1}\right\|^{2} .
\end{gathered}
$$

By (19) and (23), one concludes

$$
\theta\left\|s_{k-1}\right\|^{2} \leq s_{k-1}^{\mathrm{T}} y_{k-1} \leq L\left\|s_{k-1}\right\|^{2},
$$

where the inequality implies $\theta \leq L$.

The inequality (25) initiates

$$
s_{k-1}^{\mathrm{T}} y_{k-1}=\alpha_{k-1} d_{k-1}^{\mathrm{T}} y_{k-1}>0 .
$$


TABle 3: Summary results of EDL, MHSDL3, MHSDL4, MHSDL5, and MHSDL6 methods with respect to CPU time (sec).

\begin{tabular}{|c|c|c|c|c|c|}
\hline Test function & MHSDL3 & MHSDL4 & MHSDL5 & EDL & MHSDL6 \\
\hline Extended penalty & 29.75 & 34.11 & 31.42 & 18.30 & 24.27 \\
\hline Perturbed quadratic & 40532.66 & 24358.20 & 24947.84 & 8335.80 & 13225.80 \\
\hline Raydan 1 & 3054.67 & 1904.48 & 1692.06 & 690.91 & 1184.86 \\
\hline Raydan 2 & 6.77 & 1.58 & 1.66 & 0.31 & 0.77 \\
\hline Diagonal 1 & 7834.03 & 5106.41 & 4592.28 & 1476.89 & 486.09 \\
\hline Diagonal 2 & 885.13 & 1428.05 & 1447.02 & 2352.11 & 1513.50 \\
\hline Diagonal 3 & 13614.27 & 8416.77 & 9064.30 & 3132.02 & 1916.30 \\
\hline Hager & 586.63 & 325.75 & 333.41 & 142.06 & 198.13 \\
\hline Generalized tridiagonal 1 & 66.14 & 35.59 & 34.42 & 15.19 & 21.63 \\
\hline Extended TET & 20.50 & 78.34 & 82.94 & 41.23 & 31.45 \\
\hline Diagonal 4 & 134.53 & 77.86 & 87.88 & 30.41 & 55.34 \\
\hline Diagonal 5 & 18.06 & 134.73 & 121.09 & 0.56 & 1.84 \\
\hline Extended Himmelblau & 11.13 & 44.47 & 44.36 & 6.19 & 18.30 \\
\hline Perturbed quadratic diagonal & 91655.55 & 58226.16 & 60920.06 & 32179.38 & 36383.83 \\
\hline Quadratic QF1 & 62610.50 & 31552.48 & 28679.91 & 8832.11 & 8465.34 \\
\hline Extended quadratic penalty QP1 & 7.56 & 7.25 & 6.98 & 4.98 & 4.94 \\
\hline Extended quadratic penalty QP2 & 3814.16 & 2128.86 & 2288.55 & 671.52 & 1204.72 \\
\hline Extended quadratic exponential EP1 & 9.11 & 10.23 & 8.55 & 8.00 & 8.02 \\
\hline Extended tridiagonal 2 & 11.13 & 8.83 & 6.95 & 4.08 & 5.25 \\
\hline ARWHEAD (CUTE) & 2709.42 & 2336.92 & 2369.28 & 1266.80 & 1689.80 \\
\hline ENGVAL1 (CUTE) & 19.47 & 11.33 & 11.81 & 4.03 & 6.70 \\
\hline INDEF (CUTE) & 2.44 & 2.89 & 10.70 & 1.92 & 774.34 \\
\hline QUARTC (CUTE) & 3106.56 & 4818.58 & 4808.70 & 7138.72 & 4735.39 \\
\hline Diagonal 6 & 6.75 & 1.92 & 2.03 & 0.38 & 1.34 \\
\hline Generalized quartic & 7.16 & 11.53 & 21.05 & 7.53 & 9.78 \\
\hline Diagonal 7 & 5.98 & 8.20 & 8.28 & 4.56 & 6.25 \\
\hline Diagonal 8 & 6.17 & 8.20 & 8.08 & 4.72 & 7.69 \\
\hline Full Hessian FH3 & 30.08 & 66.45 & 79.48 & 35.77 & 43.42 \\
\hline
\end{tabular}

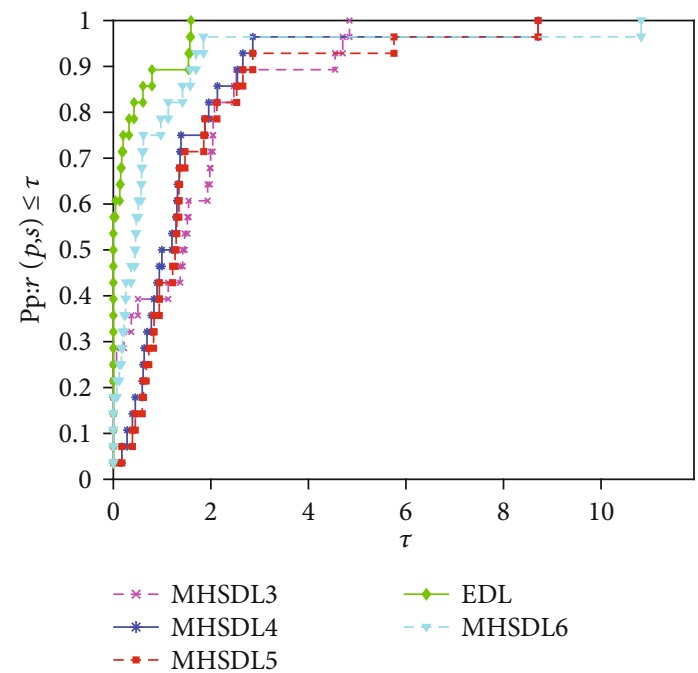

Figure 1: NI performance profile for EDL, MHSDL3, MHSDL4, MHSDL5, and MHSDL6 methods.
Taking into account $\alpha_{k-1}>0$ and the last inequality, we conclude

$$
d_{k-1}^{\mathrm{T}} y_{k-1}>0
$$

Lemma 2. [22, 23]. Let Assumption 1 be accomplished and the points $\left\{x_{k}\right\}$ be generated by the method (3)-(4). Then, it holds

$$
\sum_{k=0}^{\infty} \frac{\left\|g_{k}\right\|^{4}}{\left\|d_{k}\right\|^{2}}<+\infty
$$

Lemma 3. Consider the proposed Dai-Liao CG method, including (3), (4), and (18). If the search procedure guarantees (27), for all $k \geq 0$, then the next inequality holds

$$
g_{k}^{T} d_{k} \leq-c\left\|g_{k}\right\|^{2}
$$

for some $0 \leq c \leq 1$.

Proof. The inequality (29) will be verified by induction. In the initial situation $k=0$, one obtains $g_{0}^{\mathrm{T}} d_{0}=-\left\|g_{0}\right\|^{2}$. Since $c \leq 1$, 


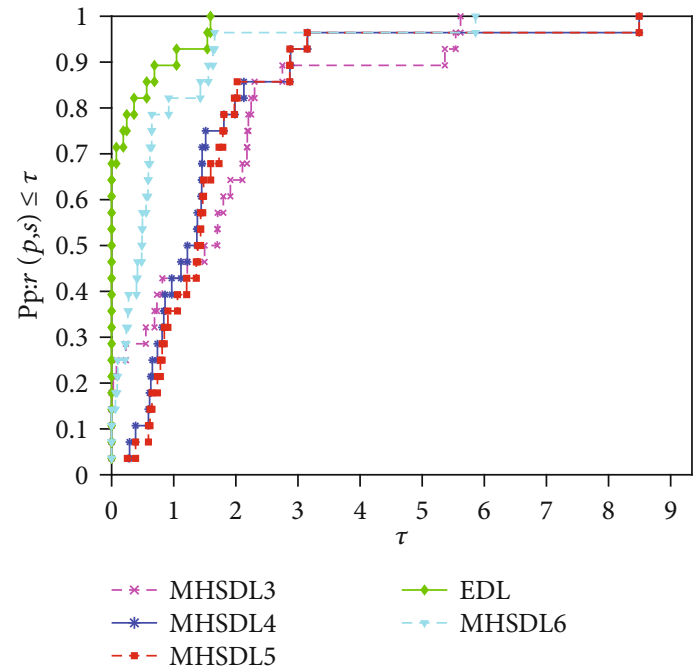

FIGURE 2: NFE performance profile for EDL, MHSDL3, MHSDL4, MHSDL5, and MHSDL6 methods.

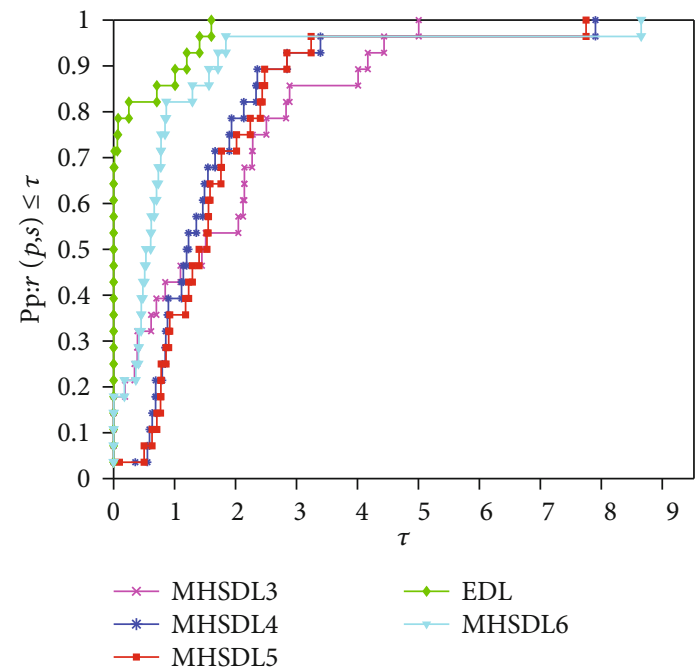

Figure 3: CPU performance profile for EDL, MHSDL3, MHSDL4, MHSDL5, and MHSDL6 methods.

obviously (29) is satisfied in the basic case. Suppose that (29) is valid for some $k \geq 1$. Taking the inner product of both the left- and right-hand sides in (4) with the vector $g_{k}^{\mathrm{T}}$, the following can be obtained:

$$
\begin{aligned}
g_{k}^{\mathrm{T}} d_{k} & =-\left\|g_{k}\right\|^{2}+\beta_{k}^{\mathrm{EDL}} g_{k}^{\mathrm{T}} d_{k-1} \\
& =-\left\|g_{k}\right\|^{2}+\left(\frac{\left\|g_{k}\right\|^{2}-\left(\left\|g_{k}\right\| /\left\|g_{k-1}\right\|\right)\left|g_{k}^{\mathrm{T}} g_{k-1}\right|}{d_{k-1}^{\mathrm{T}} y_{k-1}}-t_{k}^{*} \frac{g_{k}^{\mathrm{T}} s_{k-1}}{d_{k-1}^{\mathrm{T}} y_{k-1}}\right) g_{k}^{\mathrm{T}} d_{k-1} \\
& =-\left\|g_{k}\right\|^{2}+\frac{\left\|g_{k}\right\|^{2}-\left(\left\|g_{k}\right\| /\left\|g_{k-1}\right\|\right)\left|g_{k}^{\mathrm{T}} g_{k-1}\right|}{d_{k-1}^{\mathrm{T}} y_{k-1}} g_{k}^{\mathrm{T}} d_{k-1}-t_{k}^{*} \frac{g_{k}^{\mathrm{T}} s_{k-1}}{d_{k-1}^{\mathrm{T}} y_{k-1}} g_{k}^{\mathrm{T}} d_{k-1} \\
& =-\left\|g_{k}\right\|^{2}+\frac{\left\|g_{k}\right\|^{2}-\left(\left\|g_{k}\right\| /\left\|g_{k-1}\right\|\right)\left|g_{k}^{\mathrm{T}} g_{k-1}\right|}{d_{k-1}^{\mathrm{T}} y_{k-1}} g_{k}^{\mathrm{T}} d_{k-1}-t_{k}^{*} \frac{\alpha_{k-1}\left(g_{k}^{\mathrm{T}} d_{k-1}\right)^{2}}{d_{k-1}^{\mathrm{T}} y_{k-1}} .
\end{aligned}
$$

Using (17) in common with (27) and $\alpha_{k-1}>0$, we conclude

$$
t_{k}^{*} \frac{\alpha_{k-1}\left(g_{k}^{\mathrm{T}} d_{k-1}\right)^{2}}{d_{k-1}^{\mathrm{T}} y_{k-1}}>0
$$

Now from (30), (31), and

$$
0 \leq \beta_{k}^{\mathrm{MHS}}=\frac{\left\|g_{k}\right\|^{2}-\left(\left\|g_{k}\right\| /\left\|g_{k-1}\right\|\right)\left|g_{k}^{\mathrm{T}} g_{k-1}\right|}{d_{k-1}^{\mathrm{T}} y_{k-1}} \leq \frac{\left\|g_{k}\right\|^{2}}{\lambda\left|g_{k}^{\mathrm{T}} d_{k-1}\right|}, \quad \lambda \geq 1,
$$

it follows that

$$
\begin{aligned}
g_{k}^{\mathrm{T}} d_{k} & \leq-\left\|g_{k}\right\|^{2}+\frac{\left\|g_{k}\right\|^{2}-\left(\left\|g_{k}\right\| /\left\|g_{k-1}\right\|\right)\left|g_{k}^{\mathrm{T}} g_{k-1}\right|}{d_{k-1}^{\mathrm{T}} y_{k-1}} g_{k}^{\mathrm{T}} d_{k-1} \\
& \leq-\left\|g_{k}\right\|^{2}+\frac{\left\|g_{k}\right\|^{2}}{\lambda\left|g_{k}^{\mathrm{T}} d_{k-1}\right|}\left|g_{k}^{\mathrm{T}} d_{k-1}\right|=-\left(1-\frac{1}{\lambda}\right)\left\|g_{k}\right\|^{2} .
\end{aligned}
$$

In view of $\lambda \geq 1$, the inequality (29) is satisfied for $c=(1$ $-(1 / \lambda))$ in (33) and arbitrary $k \geq 0$.

The global convergence of the proposed EDL method is confirmed by Theorem 4 .

Theorem 4. Let Assumption 1 be true and $f$ be uniformly convex. Then, the sequence $\left\{x_{k}\right\}$ generated by (3), (4), and (18) fulfills

$$
\liminf _{k \rightarrow \infty}\left\|g_{k}\right\|=0
$$

Proof. Suppose the opposite, i.e., (34) is not true. This implies the existence of a constant $c_{1}>0$ such that

$$
\left\|g_{k}\right\| \geq c_{1}, \quad \text { for all } k \text {. }
$$

Squaring both sides of (4) implies

$$
\left\|d_{k}\right\|^{2}=\left\|g_{k}\right\|^{2}-2 \beta_{k}^{\mathrm{EDL}} g_{k}^{\mathrm{T}} d_{k-1}+\left(\beta_{k}^{\mathrm{EDL}}\right)^{2}\left\|d_{k-1}\right\|^{2} .
$$

Taking into account (18), we can get

$$
\begin{aligned}
& -2 \beta_{k}^{\mathrm{EDL}} g_{k}^{\mathrm{T}} d_{k-1}=-2\left(\frac{\left\|g_{k}\right\|^{2}-\left(\left\|g_{k}\right\| /\left\|g_{k-1}\right\|\right)\left|g_{k}^{\mathrm{T}} g_{k-1}\right|}{d_{k-1}^{\mathrm{T}} y_{k-1}}-t_{k}^{*} \frac{g_{k}^{\mathrm{T}} s_{k-1}}{d_{k-1}^{\mathrm{T}} y_{k-1}}\right) g_{k}^{\mathrm{T}} d_{k-1} \\
& =-2\left(\frac{\left\|g_{k}\right\|^{2}-\left(\left\|g_{k}\right\| /\left\|g_{k-1}\right\|\right)\left|g_{k}^{\mathrm{T}} g_{k-1}\right|}{d_{k-1}^{\mathrm{T}} y_{k-1}} g_{k}^{\mathrm{T}} d_{k-1}-t_{k}^{*} \frac{\alpha_{k-1}\left(g_{k}^{\mathrm{T}} d_{k-1}\right)^{2}}{d_{k-1}^{\mathrm{T}} y_{k-1}}\right) .
\end{aligned}
$$


Now from (31) and (32), it follows that

$$
\begin{aligned}
-2 \beta_{k}^{\mathrm{EDL}} g_{k}^{\mathrm{T}} d_{k-1} & \leq 2\left|\frac{\left\|g_{k}\right\|^{2}-\left(\left\|g_{k}\right\| /\left\|g_{k-1}\right\|\right)\left|g_{k}^{\mathrm{T}} g_{k-1}\right|}{d_{k-1}^{\mathrm{T}} y_{k-1}}\right|\left|g_{k}^{\mathrm{T}} d_{k-1}\right| \\
& \leq 2 \frac{\left\|g_{k}\right\|^{2}}{\lambda\left|g_{k}^{\mathrm{T}} d_{k-1}\right|}\left|g_{k}^{\mathrm{T}} d_{k-1}\right|=2 \frac{\left\|g_{k}\right\|^{2}}{\lambda} .
\end{aligned}
$$

Now, an application of (18) initiates

$$
\begin{aligned}
\beta_{k}^{\mathrm{EDL}} & =\frac{\left\|g_{k}\right\|^{2}-\left(\left\|g_{k}\right\| /\left\|g_{k-1}\right\|\right)\left|g_{k}^{\mathrm{T}} g_{k-1}\right|-t_{k}^{*} g_{k}^{\mathrm{T}} s_{k-1}}{d_{k-1}^{\mathrm{T}} y_{k-1}} \\
& \leq\left|\frac{g_{k}^{\mathrm{T}} g_{k}-\left(\left\|g_{k}\right\| /\left\|g_{k-1}\right\|\right)\left|g_{k}^{\mathrm{T}} g_{k-1}\right|-t_{k}^{*} g_{k}^{\mathrm{T}} s_{k-1} \mid}{d_{k-1}^{\mathrm{T}} y_{k-1}}\right| \\
& \leq \frac{\left|g_{k}^{\mathrm{T}}\left(g_{k}-\left(\left\|g_{k}\right\| /\left\|g_{k-1}\right\|\right) g_{k-1}-t_{k}^{*} s_{k-1}\right)\right|}{\theta \alpha_{k-1}\left\|d_{k-1}\right\|^{2}} \\
& =\frac{\left|g_{k}^{\mathrm{T}}\left(g_{k}-g_{k-1}+g_{k-1}-\left(\left\|g_{k}\right\| /\left\|g_{k-1}\right\|\right) g_{k-1}-t_{k}^{*} s_{k-1}\right)\right|}{\theta \alpha_{k-1}\left\|d_{k-1}\right\|^{2}} \\
& \leq \frac{\left\|g_{k}\right\|\left(\left\|g_{k}-g_{k-1}\right\|+\left\|g_{k-1}\left(1-\left(\left\|g_{k}\right\| /\left\|g_{k-1}\right\|\right)\right)\right\|+t_{k}^{*}\left\|s_{k-1}\right\|\right)}{\theta \alpha_{k-1}\left\|d_{k-1}\right\|^{2}} \\
& =\frac{\left\|g_{k}\right\|\left(\left\|g_{k}-g_{k-1}\right\|+\left|1-\left(\left\|g_{k}\right\| /\left\|g_{k-1}\right\|\right)\right|\left\|g_{k-1}\right\|+t_{k}^{*}\left\|s_{k-1}\right\|\right)}{\theta \alpha_{k-1}\left\|d_{k-1}\right\|^{2}} \\
& =\frac{\left\|g_{k}\right\|\left(\left\|g_{k}-g_{k-1}\right\|+\left|\left\|g_{k-1}\right\|-\left\|g_{k}\right\|\right|+t_{k}^{*}\left\|s_{k-1}\right\|\right)}{\theta \alpha_{k-1}\left\|d_{k-1}\right\|^{2}} \\
& \leq \frac{\left\|g_{k}\right\|\left(\left\|g_{k}-g_{k-1}\right\|+\left\|g_{k-1}-g_{k}\right\|+t_{k}^{*}\left\|s_{k-1}\right\|\right)}{\theta \alpha_{k-1}\left\|d_{k-1}\right\|^{2}} \\
& =\frac{\left\|g_{k}\right\|\left(2\left\|g_{k}-g_{k-1}\right\|+t_{k}^{*}\left\|s_{k-1}\right\|\right)}{\theta \alpha_{k-1}\left\|d_{k-1}\right\|^{2}} \leq \frac{\left\|g_{k}\right\|\left(2 L\left\|s_{k-1}\right\|+t_{k}^{*}\left\|s_{k-1}\right\|\right)}{\theta \alpha_{k-1}\left\|d_{k-1}\right\|^{2}} \\
& =\frac{\left(2 L+t_{k}^{*}\right)\left\|g_{k}\right\|\left\|s_{k-1}\right\|}{\theta \alpha_{k-1}\left\|d_{k-1}\right\|^{2}}=\frac{\left(2 L+t_{k}^{*}\right)\left\|g_{k}\right\| \alpha_{k-1}\left\|d_{k-1}\right\|}{\theta\left\|\alpha_{k-1}\right\| d_{k-1} \|^{2}} \\
& \frac{\left(2 L+g_{k-1}^{*}\right) \|}{}\left\|g_{k}\right\|
\end{aligned}
$$

Using $t_{k}^{*} \in(0,1)$ and (38) and (39) in (36), we obtain

$$
\begin{aligned}
\left\|d_{k}\right\|^{2} & \leq\left\|g_{k}\right\|^{2}+2 \frac{\left\|g_{k}\right\|^{2}}{\lambda}+\frac{\left(2 L+t_{k}^{*}\right)^{2}\left\|g_{k}\right\|^{2}}{\theta^{2}\left\|d_{k-1}\right\|^{2}}\left\|d_{k-1}\right\|^{2} \\
& =\left\|g_{k}\right\|^{2}+2 \frac{\left\|g_{k}\right\|^{2}}{\lambda}+\frac{\left(2 L+t_{\mathrm{k}}^{*}\right)^{2}}{\theta^{2}}\left\|g_{k}\right\|^{2} \\
& =\left(1+\frac{2}{\lambda}+\frac{\left(2 L+t_{k}^{*}\right)^{2}}{\theta^{2}}\right)\left\|g_{k}\right\|^{2}=\left(\frac{\lambda+2}{\lambda}+\frac{\left(2 L+t_{k}^{*}\right)^{2}}{\theta^{2}}\right)\left\|g_{k}\right\|^{2} \\
& =\frac{(\lambda+2) \theta^{2}+\lambda\left(2 L+t_{k}^{*}\right)^{2}}{\lambda \theta^{2}}\left\|g_{k}\right\|^{2} .
\end{aligned}
$$

Next, dividing both sides of (40) by $\left\|g_{k}\right\|^{4}$ and using (35), it can be concluded that

$$
\begin{gathered}
\frac{\left\|d_{k}\right\|^{2}}{\left\|g_{k}\right\|^{4}} \leq \frac{(\lambda+2) \theta^{2}+\lambda\left(2 L+t_{k}^{*}\right)^{2}}{\lambda \theta^{2}} \cdot \frac{1}{c_{1}^{2}}, \\
\frac{\left\|g_{k}\right\|^{4}}{\left\|d_{k}\right\|^{2}} \geq \frac{\lambda \theta^{2} \cdot c_{1}^{2}}{(\lambda+2) \theta^{2}+\lambda\left(2 L+t_{k}^{*}\right)^{2}} .
\end{gathered}
$$

The inequalities in (41) imply

$$
\sum_{k=0}^{\infty} \frac{\left\|g_{k}\right\|^{4}}{\left\|d_{k}\right\|^{2}} \geq \sum_{k=0}^{\infty} \frac{\lambda \theta^{2} \cdot c_{1}^{2}}{(\lambda+2) \theta^{2}+\lambda\left(2 L+t_{k}^{*}\right)^{2}}=\infty
$$

Therefore, $\left\|g_{k}\right\| \geq c_{1}$ causes a contradiction with Lemma 2.

\section{Numerical Experiments}

The implementation of the EDL method is based on Algorithm 2. This section is intended to analyze and compare the numerical results obtained by the EDL method and four variants of the MHSDL class methods (6). These variants are defined by $t \equiv t_{k 3}, t \equiv t_{k 4}, t \equiv t_{k 5}$, and $t \equiv t_{k 6}$ and denoted, respectively, as MHSDL3, MHSDL4, MHSDL5, and MHSDL6. The obtained results are not compared with the values $t_{k 1}$ and $t_{k 2}$, because in [16], the authors have already shown that $t_{k 3}$ and $t_{k 4}$ initiate better numerical performances compared to $t_{k 1}$ and $t_{k 2}$.

The codes used in the testing experiments for the above methods are written in MATLAB R2017a and executed on the Intel Core i3 $2.0 \mathrm{GHz}$ workstation with the Windows 10 operating system. Three important criteria are analyzed in each individual test case: number of iterations (NI), number of function evaluations (NFE), and processor time (CPU).

The numerical experiment is performed using 28 test functions presented in [24], where much of the problems are taken over from the CUTEr collection [25]. All methods used in the testing of an arbitrary objective function start from the same initialization $x_{0}$. Each function is tested 10 times with gradually increasing dimensions $n=100,500$, 1000, 3000, 5000, 7000, 8000, 10000, 15000, and 20000.

The uniform terminating criteria for each of the five considered algorithms (EDL, MHSDL3, MHSDL4, MHSDL5, and MHSDL6) are

$$
\begin{gathered}
\left\|g_{k}\right\| \leq \varepsilon, \\
\frac{\left|f\left(x_{k+1}\right)-f\left(x_{k}\right)\right|}{1+\left|f\left(x_{k}\right)\right|} \leq \delta,
\end{gathered}
$$

where $\varepsilon=10^{-6}$ and $\delta=10^{-16}$. The backtracking line search is based on the parameters $\omega=0.0001$ and $\varphi=0.8$ for all five algorithms. Specific parameters used only in the MHSDL6 method are defined as $C=1, v=0.26$, and $r=r_{k}=v\left\|g_{k-1}\right\|$.

Summary numerical results for EDL, MHSDL3, MHSDL4, MHSDL5, and MHSDL6 methods, executed on 28 test functions, are arranged in Tables 1-3. Tables 1-3 show the numerical outcomes corresponding to all three 
criteria (NI, NFE, and CPU) for the EDL, MHSDL3, MHSDL4, MHSDL5, and MHSDL6 methods.

We utilized the performance profile given in [26] to compare numerical results for three criteria (NI, NFE, and CPU) generated by five methods (EDL, MHSDL3, MHSDL4, MHSDL5, and MHSDL6). The upper curve of the selected performance profile corresponds to the method that shows the best performance.

Figures 1-3 plot the performance profiles for the numerical values included in Tables 1-3, respectively. Figure 1 presents the performance profiles of the NI criterion generated by the EDL, MHSDL3, MHSDL4, MHSDL5, and MHSDL6 methods. In this figure, it is noticeable that EDL, MHSDL3, MHSDL4, MHSDL5, and MHSDL6 methods solved all tested functions, wherein the EDL method shows the best performances in $57.14 \%$ of test functions compared with MHSDL3 (25.00\%), MHSDL4 (0.00\%), MHSDL5 (0.00\%), and MHSDL6 (17.86\%). From Figure 1, it is observable that the graph of the EDL method comes first to the top, which means that the EDL outperforms other considered methods with respect to the NI.

Figure 2 presents the performance profiles of the NFE of the EDL, MHSDL3, MHSDL4, MHSDL5, and MHSDL6 methods. It is observable that EDL, MHSDL3, MHSDL4, MHSDL5, and MHSDL6 generated solutions to all tested cases, and the EDL method is the best in $67.86 \%$ of the functions compared with MHSDL3 (17.86\%), MHSDL4 (0.00\%), MHSDL5 (0.00\%), and MHSDL6 (14.28\%). From Figure 2, it is observed that the EDL graph first comes to the top, which confirms that the EDL is the winner with respect to the NFE.

Figure 3 contains graphs of the performance profiles corresponding to the CPU time of the EDL, MHSDL3, MHSDL4, MHSDL5, and MHSDL6 methods. It is obvious that EDL, MHSDL3, MHSDL4, MHSDL5, and MHSDL6 solved all tested functions. Further analysis gives that the EDL method is the winner in $67.86 \%$ of the test cases compared with MHSDL3 (17.86\%), MHSDL4 (0.00\%), MHSDL5 $(0.00 \%)$, and MHSDL6 (14.28\%). Figure 3 demonstrates that the graph of the EDL method first comes to level 1, which indicates its superiority with respect to the CPU time.

From the previous analysis of the results shown in Tables 1-3 and Figures 1-3, it can be concluded that the EDL method produces superlative results in terms of all three basic metrics: NI, NFE, and CPU.

\section{Conclusion}

A novel rule which determines the value $t(k)$ of the parameter $t$ in each iteration of the Dai-Liao-type CG method is presented. The proposed expression for defining $t(k)$ is denoted by $t_{k}^{*}$. Considering $t=t_{k}^{*}$ in (6), a novel variant of the DaiLiao CG parameter $\beta_{k}^{\mathrm{EDL}}$ is defined and a novel Effective Dai-Liao (EDL) conjugate gradient method is proposed. The convergence of the EDL method is investigated, and the global convergence on a class of uniformly convex functions is established. By numerical testing, we have shown that there is a significant influence of the scalar size of $t_{k}^{*}$ on the convergence speed of the EDL method. Numerical compari- sons on large-scale unconstrained optimization test functions of different structures and complexities confirm the computational efficiency of the algorithm EDL and its superiority over the previously known DL CG variants, such as MHSDL3, MHSDL4, MHSDL5, and MHSDL6. During the testing, we tracked the number of iterations (NI), number of function evaluations (NFE), and spanned processor time (CPU) performances for each function and each method. Analysis of the obtained performance profiles introduced by Dolan and Moré revealed that the EDL method is the most efficient.

We are convinced that the obtained results will be a motivation for further research in defining new values of the parameter $t_{k}$ in the Dai-Liao CG methods. Future research would include research in finding some more efficient rules to calculate the parameter $t_{k}$ during the iterative process. We hope that our proposal of the new expression for defining the parameter $t$ will initiate further research in that direction. It is evident that finding novel approaches in defining different values of $t$ and the conjugate gradient parameter $\beta_{k}$ is an inexhaustible topic for scientific research, and our approach is only one possible direction in this research.

\section{Data Availability}

Data will be provided on request to the first author.

\section{Conflicts of Interest}

The authors declare no conflict of interest.

\section{Acknowledgments}

The research was supported by the National Natural Science Foundation of China (Grant Nos. 11971142, 11871202, $61673169,11701176,11626101$, and 11601485).

\section{References}

[1] Y. -H. Dai and L. -Z. Liao, "New conjugacy conditions and related nonlinear conjugate gradient methods," Applied Mathematics and Optimization, vol. 43, no. 1, pp. 87-101, 2001.

[2] Y. Cheng, Q. Mou, X. Pan, and S. Yao, "A sufficient descent conjugate gradient method and its global convergence," Optimization Methods and Software, vol. 31, no. 3, pp. 577-590, 2016.

[3] I. E. Livieris and P. Pintelas, "A descent Dai-Liao conjugate gradient method based on a modified secant equation and its global convergence," ISRN Computational Mathematics, vol. 2012, Article ID 435495, 8 pages, 2012.

[4] M. R. Peyghami, H. Ahmadzadeh, and A. Fazli, "A new class of efficient and globally convergent conjugate gradient methods in the Dai-Liao family," Optimization Methods and Software, vol. 30, no. 4, pp. 843-863, 2015.

[5] H. Yabe and M. Takano, "Global convergence properties of nonlinear conjugate gradient methods with modified secant condition," Computational Optimization and Applications, vol. 28, no. 2, pp. 203-225, 2004. 
[6] S. Yao and B. Qin, "A hybrid of DL and WYL nonlinear conjugate gradient methods," Abstract and Applied Analysis, vol. 2014, Article ID 279891, 9 pages, 2014.

[7] S. Yao, X. Lu, and Z. Wei, “A conjugate gradient method with global convergence for large-scale unconstrained optimization problems," Journal of Applied Mathematics, vol. 2013, Article ID 730454, 9 pages, 2013.

[8] Y. Zheng and B. Zheng, "Two new Dai-Liao-type conjugate gradient methods for unconstrained optimization problems," Journal of Optimization Theory and Applications, vol. 175, no. 2, pp. 502-509, 2017.

[9] W. Zhou and L. Zhang, "A nonlinear conjugate gradient method based on the MBFGS secant condition," Optimization Methods and Software, vol. 21, no. 5, pp. 707-714, 2006.

[10] W. Hu, J. Wu, and G. Yuan, "Some modified Hestenes-Stiefel conjugate gradient algorithms with application in image restoration," Applied Numerical Mathematics, vol. 158, pp. 360376, 2020.

[11] G. Yuan, T. Li, and W. Hu, “A conjugate gradient algorithm for large-scale nonlinear equations and image restoration problems," Applied Numerical Mathematics, vol. 147, pp. 129-141, 2020.

[12] N. Andrei, "Open problems in nonlinear conjugate gradient algorithms for unconstrained optimization," Bulletin of the Malaysian Mathematical Sciences Society, vol. 34, no. 2, pp. 319-330, 2011.

[13] W. W. Hager and H. Zhang, "A new conjugate gradient method with guaranteed descent and an efficient line search," SIAM Journal on Optimization, vol. 16, no. 1, pp. 170-192, 2005.

[14] W. W. Hager and H. Zhang, "Algorithm 851," ACM Transactions on Mathematical Software, vol. 32, no. 1, pp. 113-137, 2006.

[15] Y. -H. Dai and C. -X. Kou, "A nonlinear conjugate gradient algorithm with an optimal property and an improved Wolfe line search," SIAM Journal on Optimization, vol. 23, no. 1, pp. 296-320, 2013.

[16] S. Babaie-Kafaki and R. Ghanbari, "The Dai-Liao nonlinear conjugate gradient method with optimal parameter choices," European Journal of Operational Research, vol. 234, no. 3, pp. 625-630, 2014.

[17] N. Andrei, "A Dai-Liao conjugate gradient algorithm with clustering of eigenvalues," Numerical Algorithms, vol. 77, no. 4, pp. 1273-1282, 2018.

[18] M. Lotfi and S. M. Hosseini, "An efficient Dai-Liao type conjugate gradient method by reformulating the CG parameter in the search direction equation," Journal of Computational and Applied Mathematics, vol. 371, article 112708, 2020.

[19] X. Li and Q. Ruan, "A modified PRP conjugate gradient algorithm with trust region for optimization problems," Numerical Functional Analysis and Optimization, vol. 32, no. 5, pp. 496506, 2011.

[20] N. Andrei, "An acceleration of gradient descent algorithm with backtracking for unconstrained optimization," Numerical Algorithms, vol. 42, no. 1, pp. 63-73, 2006.

[21] P. S. Stanimirovic and M. B. Miladinovic, "Accelerated gradient descent methods with line search," Numerical Algorithms, vol. 54, no. 4, pp. 503-520, 2010.

[22] W. Cheng, "A two-term PRP-based descent method," Numerical Functional Analysis and Optimization, vol. 28, no. 11-12, pp. 1217-1230, 2007.
[23] G. Zoutendijk, "Nonlinear programming, computational methods," in Integer and Nonlinear Programming, North-Holland, J. Abadie, Ed., pp. 37-86, North-Holland, Amsterdam, 1970.

[24] N. Andrei, "An unconstrained optimization test functions collection," Advanced Modeling and Optimization, vol. 10, no. 1, pp. 147-161, 2008.

[25] I. Bongartz, A. R. Conn, N. Gould, and P. L. Toint, "CUTE: constrained and unconstrained testing environments," $A C M$ Transactions on Mathematical Software, vol. 21, no. 1, pp. 123-160, 1995.

[26] E. D. Dolan and J. J. Moré, "Benchmarking optimization software with performance profiles," Mathematical Programming, vol. 91, no. 2, pp. 201-213, 2002. 